\title{
Lack-of-fit Testing for Polynomial Regression Models Without Replications
}

\author{
Maha A. Omair ${ }^{1}$, Abdullah A. Al-Shiha ${ }^{1}$ \& Ruba A. Alyafi ${ }^{1}$ \\ ${ }^{1}$ Department of Statistics and Operations Research, College of Sciences, King Saud University, Saudi Arabia \\ Correspondence: Maha A. Omair, Department of Statistics and Operations Research, College of Sciences, King \\ Saud University, Saudi Arabia.
}

Received: June 24, 2019 Accepted: July 31, 2019 Online Published: August 14, 2019

doi:10.5539/ijsp.v8n5p49

URL: https://doi.org/10.5539/ijsp.v8n5p49

\begin{abstract}
Parametric and non-parametric approaches are developed to test the adequacy of the polynomial model $Y=\beta_{\circ}+\sum_{i=1}^{p} \beta_{j} X^{j}+\varepsilon$ when there is no replication in the values of the independent variable. The proposed tests avoid partitioning of the sample space of the continuous covariate. This paper suggests three tests based on the following concept: if the model is appropriate for a selected application, then the error component $\varepsilon_{1}, \varepsilon_{2}, \ldots, \varepsilon_{n}$ is a random sample with zero mean and constant variance. Simulation results are provided to illustrate the power and size of the proposed tests. An example is used to illustrate the methodologies. These tests are also compared with the classical lack-of-fit test to demonstrate their advantage.
\end{abstract}

Keywords: lack-of-fit test, regression model, replication, non parametric test

\section{Introduction}

A very important aspect in regression analysis is testing the adequacy of the proposed model. The classical lack-of-fit test, which was given by Fisher (1922), is appropriate for a model with replication. In most empirical and some experimental data, replication is unavailable. Several approaches are available for testing the adequacy of such models. Christensen (1989) introduced the concept of lack of fit between or within clusters, or a mixture of these two pure types, for near replicates. Su and Wei (1991) showed by example that such tests based on different partitions of the regression variable space may lead to different conclusions on the adequacy of a proposed model. Christensen (2003) noted that a small value of the F-statistic does not indicate a lack of fit in the proposed model, but in the combination of these two pure types, and this can be extremely difficult to detect. Miller et al. (1998, 1999), Miller and Neil (2007) presented a lack-of-fit test based on families of groupings of the observation, using the test given by Christensen (1989, 1991), and those given by Khuri (1985) and Levy and Neill (1990).

Nearly all studies involve dividing the data across the predictor spaces, assumption of a grouping structure, and estimation of the pure error to obtain an approximated F-statistic. This study proposes a simple approach that avoids partitioning of the continuous variable, to test the adequacy of the model when replication is not available. Alyafi et al. (2017) examined the lack of fit of a polynomial model by applying the Durbin-Watson test on the residuals.

In Section 2, three tests are presented. All these tests are based on the following concept: if the model is appropriate for a selected application, then the error component $\varepsilon_{i}(i=1,2, \ldots, n)$ is a random sample with zero mean and variance $\sigma^{2}$. For the sake of simplicity, we can assume approximately that $e_{i}(i=1,2, \ldots, n)$ is a random sample from a normal distribution with zero mean if the model is appropriate.

In the case of one independent variable, we assume the model as

$$
Y_{i}=\sum_{j=0}^{p} \beta_{j} X_{i}^{j}+\varepsilon_{i} ; i=1,2, \ldots, n
$$

while the underlying true model has the form

$$
Y_{i}=\sum_{j=0}^{p} \beta_{j+1} X_{i}^{j+1}+\varepsilon_{i} ; i=1,2, \ldots, n
$$

where $X_{i}$ is a known constant, the value of the independent variable in the ith trial.

Let $X_{(1)}<X_{(2)} \ldots \ldots<X_{(n)}$ be the ordered values of the independent variable.

Let $v_{i}=e_{[i]}$ be the residual associated with the ordered value $X_{(i)}$.

Let $p=1,2$, and 3 be the degree of the independent variable. 
We also considered standardized residuals instead of the classic residuals as standardized residuals allow the residuals to be compared on a standard scale.

The tests evaluate the adequacy of the proposed model by detecting randomness of the sample $v_{1}, v_{2}, \ldots, v_{n}$, using the following hypotheses:

$$
\begin{aligned}
& H_{0}: \quad v_{1}, v_{2}, \ldots, v_{n} \text { is a random sample. (The model is adequate) } \\
& H_{1}: \quad v_{1}, v_{2}, \ldots, v_{n} \text { is not a random sample. (The model is not adequate) }
\end{aligned}
$$

Simulations are provided in the subsequent section. Moreover, conclusions and comparisons between the powers of the three proposed tests and the Durbin-Watson approach discussed in Alyafi et al. (2017) are illustrated in the section "Comparison and Conclusions."

Furthermore, in the section "Illustrative Example," an applied example by Neter, Kutner, and Wasserman (1985) is used to illustrate the test methodologies and compare them with the classical lack-of-fit test.

\section{Method}

\section{Test 1}

A new non-parametric test is presented to test the lack of fit of the regression model (1) when replication is not available. To test the randomness of the residuals $v_{1}, v_{2}, \ldots, v_{n}$ associated with the ordered $X_{(i)}$, we define the random variables

$$
U_{i}=I_{(-\infty, 0)}\left(v_{i} v_{i+1}\right)=\left\{\begin{array}{l}
1 ; v_{i} v_{i+1}<0 \Leftrightarrow \operatorname{sign}\left(v_{i}\right) \neq \operatorname{sign}\left(v_{i+1}\right) \\
0 ; v_{i} v_{i+1}>0 \Leftrightarrow \operatorname{sign}\left(v_{i}\right)=\operatorname{sign}\left(v_{i+1}\right)
\end{array} ; i=1,2, \ldots n\right.
$$

where $U=\sum_{i=1}^{n-1} U_{i}$.

Under the null hypothesis $H_{0}$ (the model is adequate), $v_{1}, v_{2}, \ldots, v_{n}$ are approximately i.i.d. $N\left(0, \sigma_{v}^{2}\right)$, i.e., $\boldsymbol{V} \sim N_{n}\left(\mathbf{0}, \sigma_{v}^{2} \boldsymbol{I}_{n}\right)$, where the random vector $\boldsymbol{V}^{\prime}$ is $\left(v_{1}, v_{2}, \ldots, v_{n}\right)$.

We will introduce four theorems below about the random variable $U_{i}$ defined above. Their proofs are in Appendix A.

Theorem 1:

The random variable $U_{i}$ has a Bernoulli distribution with the parameter $p_{i}$ for each $i=1,2, \ldots, n-1$. That is, $U_{i} \sim \operatorname{Binomial}\left(1, p_{i}\right)$.

Theorem 2:

Under $H_{0}, U_{i} \sim \operatorname{Binomial}(1,0.5)$, for each $i=1,2, \ldots, n-1$.

Theorem 3:

Under $H_{0}, U_{1}, U_{2}, \ldots, U_{n-1}$ are independent.

Theorem 4:

Under $H_{0}, U=\sum_{i=1}^{n-1} U_{i} \sim \operatorname{Binomial}(n-1,0.5)$.

Based on the definition of the statistic $U$, large and small values of $U$ indicate that the sample is not random.

Therefore, for a given $\alpha$, we reject $\mathrm{H}_{0}$ if $\mathrm{P}\left(U \leq u_{0}\right) \leq \alpha / 2$ or $\mathrm{P}\left(U \geq u_{0}\right) \leq \alpha / 2$, where $u_{0}=\sum_{i=1}^{n-1} u_{i}$.

For a large sample size, we considered the binomial approximation to normal where $U \approx N(n p, n p q)$ i.e., $U \approx$ $N\left(\frac{n-1}{2}, \frac{n-1}{4}\right)$.

\section{Test 2}

This test uses the Wald-Wolfowitz [13] runs test technique to test whether there is any trend with the ordered residuals $v_{1}, v_{2}, \ldots, v_{n}$. Depending on the runs of the signs in the ordered residuals, the probabilities of the number of runs $\mathrm{P}(\mathrm{T} \leq \mathrm{r})$ and $\mathrm{P}(\mathrm{T} \geq \mathrm{r})$ are calculated, where $\mathrm{T}$ is the number of runs and $\mathrm{r}$ is the observed value of $\mathrm{t}$. The test hypotheses are $\mathrm{H}_{0}$ : The sequence is random against $\mathrm{H}_{1}$ : The sequence is not random.

$\mathrm{H}_{0}$ is rejected at level $\alpha$ if $\mathrm{P}(\mathrm{T} \leq \mathrm{r}) \leq \alpha / 2$ or $\mathrm{P}(\mathrm{T} \geq \mathrm{r}) \leq \alpha / 2$.

The probability distribution of the number of runs under $\mathrm{H}_{0}$ is 


$$
P[T=r]=\left\{\begin{array}{c}
\frac{2\left(\begin{array}{c}
A-1 \\
\frac{r}{2}-1
\end{array}\right)\left(\begin{array}{l}
B-1 \\
\frac{r}{2}-1
\end{array}\right)}{\left(\begin{array}{c}
A+B \\
A
\end{array}\right)} \\
\text {, for even } r \\
\frac{\left(\begin{array}{c}
A-1 \\
(r-1) / 2
\end{array}\right)\left(\begin{array}{c}
B-1 \\
(r-3) / 2
\end{array}\right)+\left(\begin{array}{c}
A-1 \\
(r-3) / 2
\end{array}\right)\left(\begin{array}{c}
B-1 \\
(r-1) / 2
\end{array}\right)}{\left(\begin{array}{c}
A+B \\
A
\end{array}\right)}, \text { for odd } r,
\end{array}\right.
$$

where $\mathrm{A}$ and $\mathrm{B}$ correspond to the number of positive and negative runs, respectively.

For large sample sizes, where the numbers A and B are larger than ten, the normal approximation is adequate, where $\mathrm{T} \approx N(E(T), V(T))$ and $\quad E(T)=\frac{2 A B}{A+B}+1, V(T)=\frac{2 A B(2 A B-A-B)}{(A+B)^{2}(A+B-1)}$.

\section{Test 3}

Test 3 tests the lack of fit of the regression model (1) by introducing the following regression model:

$$
v_{k}=\gamma_{0}+\sum_{j=1}^{q} \gamma_{j} k^{j}+\varepsilon_{k} ; \quad k=1,2, \ldots, n ; q=1
$$

where $v_{k}$ 's are the residuals associated with the ordered $\mathrm{x}$ values from Model (1).

The value of $q$ in model (5) depends on the degree of the independent variable in the true model i.e., q takes the value of the power of the independent variable plus one, if the power of the dependent variable is even; if the power is odd, $\mathrm{q}$ equals the power plus two. Here, we wish q to be an odd number as the time $k$ with an odd power will be more sensitive in detecting true and false models.

The classical Fisher's test statistic $\mathrm{F}$ is used on model (5) to test $H_{0}: \gamma_{1}=\gamma_{2}=\ldots=\gamma_{q}=0$ against $H_{1}: \gamma_{j} \neq 0$ for some $j=1,2, \ldots, q$.

\section{Simulation Results}

Simulations were performed to compare the power and size of the proposed tests. In particular, the adequacy of model (1) was tested when the observations were truly generated from model (2) for different parameter values $\beta_{j}=0.5,1,5$ for $j=1,2,3$. For each simulation, the independent variable $\mathrm{X}$ was generated from random real numbers ranging from 0 to $\mathrm{c}$ (where $c=1,5,20$ and 50). Errors were generated independently from $\mathrm{N}\left(\beta_{0}+\sum_{j=1}^{p} \beta_{j} X_{i}^{j}, \sigma^{2}\right)$ where $i=1,2, \ldots, n, \sigma^{2}$ takes the values $0.1,0.5,1$ and 5, the dependent variable $\mathrm{Y}$ was fitted to both models (1) and (2), and 10000 simulation datasets were generated according to the previous specifications with $\mathrm{n}=10,30$, and 100 . The percentage of rejection of the false model (model (1)) was used to calculate the empirical power of the test, and the percentage of rejection of the true model (model (2)) was used to calculate the empirical size of the test. The simulation was performed using the software Mathematica 8 , where $\alpha$, the significant level, takes the values $0.01,0.05$, and 0.1 .

As indicated in "Introduction," all the three tests depend on the randomness of the residuals $v_{1}, v_{2}, \ldots, v_{n}$.

These residuals are the residuals associated with the ordered $X_{i}$. The residuals $v_{1}, v_{2}, \ldots, v_{n}$ are calculated for both models (1) and (2).

Through simulation, the test statistics $\mathrm{U}_{0}, \mathrm{~T}_{0}$, and $\mathrm{F}_{0}$ were calculated for model (1) for all the three tests. Similarly, the test statistics $\mathrm{U}_{1}, \mathrm{~T}_{1}$, and $\mathrm{F}_{1}$ were calculated for model (2). Depending on the rejection rule, the approximate power and size of the tests were computed for specified $\alpha$ levels.

The simulated results of power and size for testing the adequacy of model (1) for different values of $p$ are shown in Figure 1. In particular, this figure shows the empirical power and size of all the three tests when the observations were generated from model (2). The results of the Durbin-Watson approach (test 4) in Alyafi et al. (2017) are also illustrated in Figure 1.

Note: Test 4 is not applicable when $\mathrm{p}=0$ as the Durbin-Watson test requires at least one independent variable.

$$
\text { "Size of the test" }
$$

"Power of the test" 


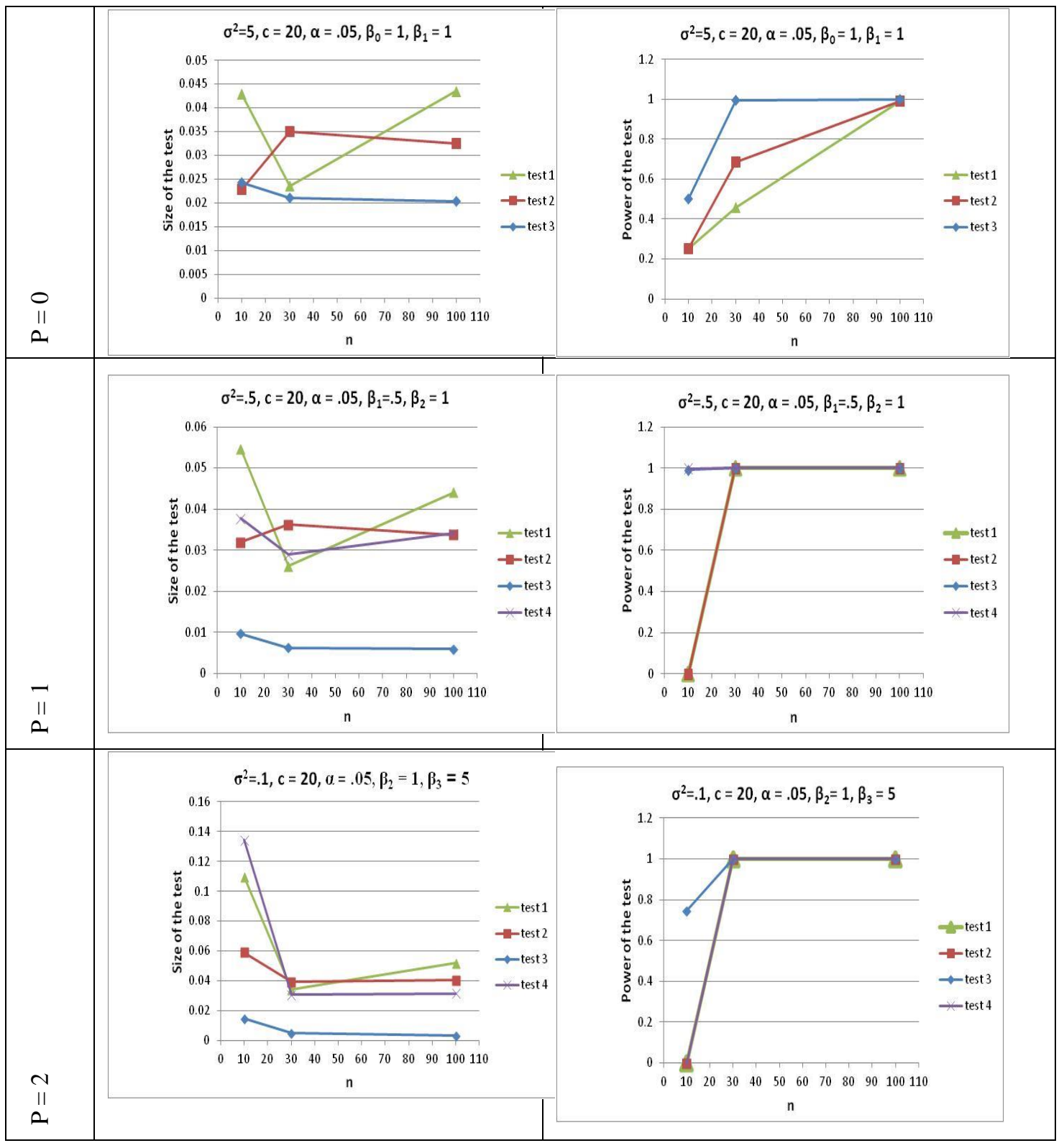

Figure 1. Comparison of the empirical size and power of the tests for the mentioned cases 


\section{Comparison and Conclusion}

Throughout the simulations, all the tests provided a very strong empirical power of almost 1 for $n=30$ and $n=100$. In addition, the empirical size of the test was close to the assumed $\alpha$.

The empirical size of test 1 was closer to the assumed $\alpha$ than that of any other test. Test 3 provided a very strong empirical power - almost one - for all sample sizes and a low empirical size for all sample sizes.

For test 1 , when $\mathrm{n}=10$, the empirical power of the test was almost zero. From the simulations, we observed that $\mathrm{u}_{0}$ takes the value 2 in most cases. Hence, after calculating $\mathrm{P}\left(\mathrm{U} \leq \mathrm{u}_{0}\right), \mathrm{P}\left(\mathrm{U} \geq \mathrm{u}_{0}\right) \mathrm{H}_{0}$ was always accepted for the false model for any given $\alpha$. We investigated further and observed that, for $n \geq 16$, the performance of this test improved significantly. For test 2, considering all the possible run values when $\mathrm{n}=10, \mathrm{H}_{0}$ was almost never rejected, which results in an empirical power equal to almost zero. As shown in Figure 1, when $p=0$, test 4 is not applicable, as the Durbin-Watson tables are provided only when a constant term in the regression model and at least one independent variable exist.

As expected, the simulated power for testing model (1) increases as $n$ increases and decreases as $\sigma^{2}$ increases.

Overall, all our proposed tests showed very promising results. However, some tests are preferred over others in some specific cases.

\section{Illustrative Example}

A dataset will be analyzed here to illustrate the methods of our four tests. The data of interest are given by Neter, Kutner and Wasserman (1985) in Applied Linear Statistical Models, as shown in Table 1. The data include promotional expenditure $\mathrm{X}$ and demand for a specific firm's product $\mathrm{Y}$.

Table 1. Promotional expenditure (X) and the demand for a specific firm's product $(\mathrm{Y})$ in a territory (X in thousand dollars, $\mathrm{Y}$ in thousand units)

\begin{tabular}{lllllllllllllll}
\hline $\begin{array}{l}\text { Perio } \\
\mathrm{d}\end{array}$ & 1 & 2 & 3 & 4 & 5 & 6 & 7 & 8 & 9 & 10 & 11 & 12 & 13 & 14 \\
\hline $\mathrm{X}$ & 17 & 15 & 25 & 10 & 18 & 15 & 20 & 25 & 17 & 13 & 20 & 23 & 25 & 16 \\
& 56.1 & 54. & 55.2 & 52.5 & 56.2 & 55.9 & 55.5 & 54.3 & 55.1 & 54.2 & 55.7 & 55.6 & 54.9 & 55.0 \\
$\mathrm{Y}$ & 5 & 5 & 7 & 4 & 3 & 7 & 5 & 2 & 4 & 8 & 8 & 5 & 6 & 6 \\
\hline
\end{tabular}

First, we will fit the linear model

$$
\widehat{Y}=53.7+.0778 x
$$

Figures 2 and 3 suggest nonlinearity. From the figures below, we observe that the relation between the firm's product and the promotional expenditure is quadratic.

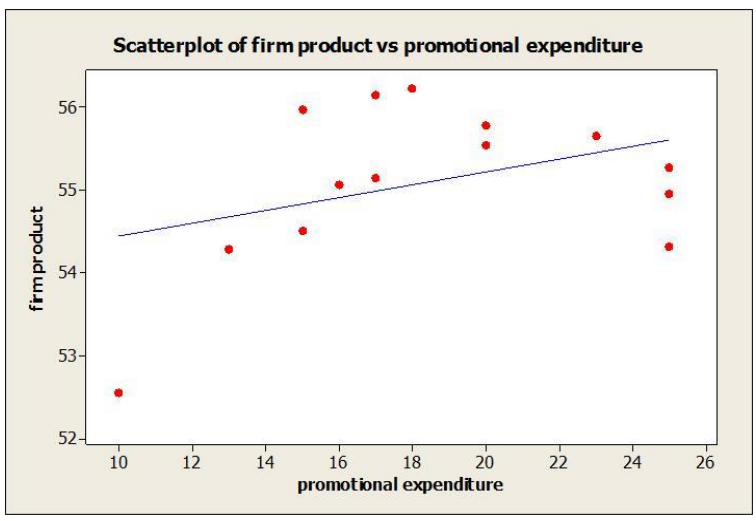

Figure 2. Scatter plot for product example illustrating nonlinearity function

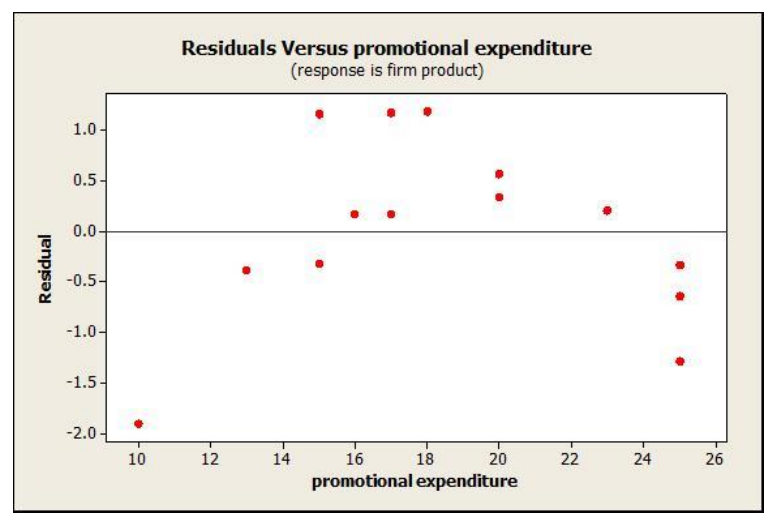

Figure 3. Residuals plot for product example illustrating nonlinearity function 
Now, we will consider fitting the quadratic regression model.

The fitted model is

$$
\hat{Y}=42.1+1.41 x-0.0358 x^{2}
$$

For the quadratic model, Figures 4 and 5 suggest that the quadratic function is a good fit.

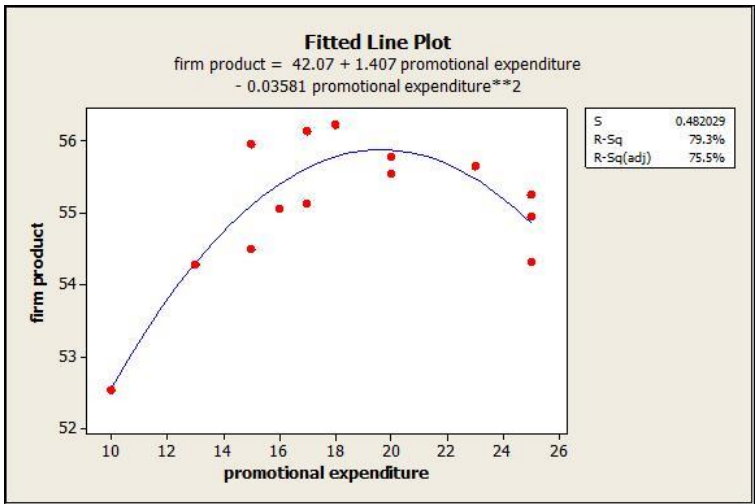

Figure 4. Fitted quadratic-order polynomial regression function for product example

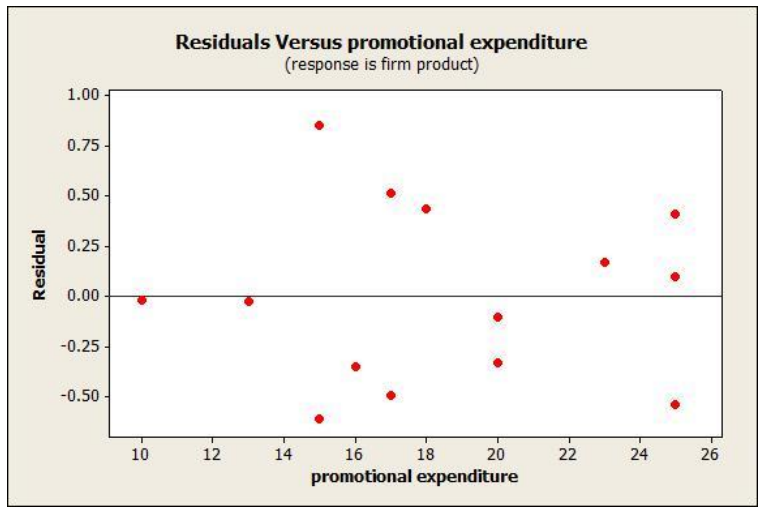

Figure 5. Residuals plot against $\mathrm{x}$ for product example

To show the promising performance of the four suggested tests, we test the adequacy of both the linear and quadratic models using all four proposed tests in the Section 2.

\section{a) Test 1:}

To test the linear model, we fit $v_{1}, v_{2}, \ldots, v_{14}$ obtained from the linear model (6).

We defined a random variable $\mathrm{U}$ that depends on the sign of the residuals $v_{1}, v_{2}, \ldots, v_{14}$

Using (3), we have $u_{0}=2, P(U \leq 2)=0.0112$ and $P(U \geq 2)=0.9983$.

For $\alpha=0.01$, we cannot reject the linear model, i.e., the linear model is appropriate.

For $\alpha=0.05$ and $\alpha=0.1$, the linear model is not appropriate.

To test the quadratic model, we fit $v_{1}, v_{2}, \ldots, v_{14}$ obtained from the quadratic model (7).

We defined a random variable $\mathrm{U}$ that depends on the sign of the residuals $v_{1}, v_{2}, \ldots, v_{14}$

Using (3), we have $u_{0}=7, P(U \leq 7)=0.7095$ and $P(U \geq 7)=0.5$.

For $\alpha=0.01, \alpha=0.05$, and $\alpha=0.1$, the quadratic model is appropriate.

Comment: As expected, we did not reject the linear model for $\alpha=0.01$ even though it is the false model. This result is consistent with our conclusion Section 4.

\section{b) Test 2:}

To test the linear model, we fit $v_{1}, v_{2}, \ldots, v_{14}$ obtained from the linear model (6). This test depends on the number of runs of the signs in the residuals $v_{1}, v_{2}, \ldots, v_{14}$. The number of positive signs $=8$, the number of negative signs $=6$, and the number of runs $=3$.

Using (4), we obtain $P(T \leq 3)=0.0047$ and $P(T \geq 3)=0.9933$.

For $\alpha=0.01, \alpha=0.05$, and $\alpha=0.1$, the linear model is not appropriate.

To test the quadratic model, we fit $v_{1}, v_{2}, \ldots, v_{14}$ obtained from the quadratic model (7). This test depends on the number of runs of the signs in the residuals $v_{1}, v_{2}, \ldots, v_{14}$. The number of positive signs $=6$, the number of negative signs $=8$, and the number of runs $=8$.

Using (4), we obtain $P(T \leq 8)=0.6457$ and $P(T \geq 8)=0.5874$.

For $\alpha=0.01, \alpha=0.05$, and $\alpha=0.1$, the quadratic model is appropriate.

\section{c) Test 3 :}

To test the linear model, we fit $v_{1}, v_{2}, \ldots, v_{14}$ obtained from the linear model (6) to model (5) with $\mathrm{q}=3$. $\hat{v}_{k}=-3.0852+1.4901 k^{1}+-0.1722 k^{2}+0.0056 k^{3} ;$ where $\mathrm{k}=1,2,3, \ldots, 14$. 
The $p$-value $=0.0054$, suggesting that the linear model is not appropriate for all $\alpha$.

To test the quadratic model, we fit $v_{1}, v_{2}, \ldots, v_{14}$ obtained from the quadratic model (7) to model (5) with $\mathrm{q}=3$.

$\hat{v}_{k}=-0.3480+0.2123 k^{1}+-0.0343 k^{2}+0.0016 k^{3} ;$ where $\mathrm{k}=1,2,3, \ldots, 14$.

The $p$-value $=0.9242$, suggesting that the quadratic model is appropriate for all $\alpha$.

\section{d) Test 4:}

To test the linear model, we fit $v_{1}, v_{2}, \ldots, v_{14}$ obtained from the linear model (6).

We test the serial correlation between the residuals $v_{1}, v_{2}, \ldots, v_{14}$.

Based on the Durbin-Watson test statistic, DW $=0.9461$ and $4-\mathrm{DW}=3.0539$.

At $\alpha=0.05 \Rightarrow d l=0.91523$ and $d u=1.2086$.

At $\alpha=0.1 \Rightarrow d l=1.04495$ and $d u=1.3502$.

For $\quad \alpha=0.05 \Rightarrow$ inconclusive .

For $\alpha=0.1 \Rightarrow$ the linear model is not appropriate.

To test the quadratic model, we fit $v_{1}, v_{2}, \ldots, v_{14}$ obtained from the quadratic model (7).

We test the serial correlation between the residuals $v_{1}, v_{2}, \ldots, v_{14}$.

Based on the Durbin-Watson test statistic, DW $=2.6353$ and $\quad$ 4-DW $=1.3647$.

At $\alpha=0.05 \Rightarrow d l=0.7862$ and $d u=1.4092$.

At $\alpha=0.1 \Rightarrow d l=0.9054$ and $d u=1.5507$.

For $\alpha=0.05$ and $\alpha=0.1 \Rightarrow$ inconclusive .

Comment: As the sample size is small, i.e., $\mathrm{n}=14$, predictably, the test is said to be inconclusive.

\section{Classical Lack-of-fit Test}

Testing the adequacy of the linear model (6), we have the F-statistic $=2.91$ and p-value $=0.128$, suggesting that the linear model is appropriate for all $\alpha$.

Testing the adequacy of the quadratic model (7), we have the F-statistic $=0.19$ and p-value $=0.967$, suggesting that the quadratic model is appropriate for all $\alpha$.

To confirm the powerfulness and accuracy of our tests, we consider $\alpha=0.05$ in our proposed example. All the tests provided the correct decision regarding models (6) and (7), except for test 4 , which was inconclusive. In contrast, the lack-of-fit test provided an incorrect decision for model (6) but a correct one for model (7).

\section{Acknowledgments}

The authors thank the Deanship of Scientific Research and RSSU at King Saud University for their technical support.

\section{References}

Alyafi, R. A., Omair, M. A., \& Al-Shiha, A. A. (2017). Durban Watson for Testing the Lack-of-Fit of Polynomial Regression Models without Replications. Journal of Global Research in Mathematical Archives (JGRMA), 4(11), 165-172.

Christensen, R. (1991). Small-sample characterizations of near replicate lack-of-fit tests. Journal of the American Statistical Association, 86(415), 752-756. https://doi.org/10.1080/01621459.1991.10475105

Christensen, R. (1989). Lack-of-fit tests based on near or exact replicates. The Annals of Statistics, 673-683. https://doi.org/10.1214/aos/1176347133

$\mathrm{Su}$, J. Q., \& Wei, L. J. (1991). A lack-of-fit test for the mean function in a generalized linear model. Journal of the American Statistical Association, 86(414), 420-426. https://doi.org/10.1080/01621459.1991.10475059

Christensen, R. (2003). Significantly insignificant F tests. The American Statistician, 57(1), 27-32. https://doi.org/10.1198/0003130031108

Fisher, R. A. (1922). The goodness of fit of regression formulae, and the distribution of regression coefficients. Journal of the Royal Statistical Society, 85(4), 597-612. https://doi.org/10.2307/2341124

Khuri, A. I. (1985). A test for lack of fit of a linear multiresponse model. Technometrics, 27(3), 213-218. https://doi.org/10.1080/00401706.1985.10488045 
Levy, M. S., \& Neill, J. W. (1990). Testing for lack of fit in linear multiresponse models based on exact or near replicates. Communications in Statistics-Theory and Methods, 19(6), 1987-2002. https://doi.org/10.1080/03610929008830304

Miller, F. R., \& Neill, J. W. (2008). General lack of fit tests based on families of groupings. Journal of Statistical Planning and Inference, 138(8), 2433-2449. https://doi.org/10.1016/j.jspi.2007.10.025

Miller, F. R., Neill, J. W., \& Sherfey, B. W. (1998). Maximin clusters for near-replicate regression lack of fit tests. The Annals of Statistics, 26(4), 1411-1433. https://doi.org/10.1214/aos/1024691249

Miller, F. R., Neill, J. W., \& Sherfey, B. W. (1999). Implementation of a maximin power clustering criterion to select near replicates for regression lack-of-fit tests. Journal of the American Statistical Association, 94(446), 610-620. https://doi.org/10.1080/01621459.1999.10474155

Neter, J., Kutner, M. H., Nachtsheim, C. J., \& Wasserman, W. (1996). Applied linear statistical models . Chicago: Irwin.

Wald, A., \& Wolfowitz, J. (1940). On a test whether two samples are from the same population. The Annals of Mathematical Statistics, 11(2), 147-162. https://doi.org/10.1214/aoms/1177731909

\section{Appendix A}

\section{Theorem 1:}

The random variable $U_{i}$ has a Bernoulli distribution with the parameter $p_{i}$ for each $i=1,2, \ldots, n-1$. That is, $U_{i} \sim \operatorname{Binomial}\left(1, p_{i}\right)$.

\section{Proof:}

The random variable $U_{i}$ is a binary variable, with $p_{i}=P\left(U_{i}=1\right)=P\left(v_{i} v_{i+1}<0\right)$ and $1-p_{i}=P\left(U_{i}=0\right)=$ $P\left(v_{i} v_{i+1} \geq 0\right)$. Hence, $U_{i} \sim \operatorname{Binomial}\left(1, p_{i}\right)$.

\section{Theorem 2:}

Under $H_{0}, U_{i} \sim \operatorname{Binomial}(1,0.5)$, for each $i=1,2, \ldots, n-1$.

Proof:

We define the random vector $\boldsymbol{V}_{i, i+1}=\left(\begin{array}{c}v_{i} \\ v_{i+1}\end{array}\right)$.

Under $H_{0}$, we have $\boldsymbol{V}_{i, i+1} \sim N_{2}\left(\mathbf{0}, \sigma_{v}^{2} \boldsymbol{I}_{2}\right)$.

Now,

$$
\begin{aligned}
& p_{i}=P\left(U_{i}=1\right)=P\left(v_{i} v_{i+1}<0\right) \\
& =P\left(v_{i}<0, v_{i+1}>0\right)+P\left(v_{i}>0, v_{i+1}<0\right) \\
& =P\left(v_{i}<0\right) P\left(v_{i+1}>0\right)+P\left(v_{i}>0\right) P\left(v_{i+1}<0\right)=2(0.5)(0.5) \\
& =0.5
\end{aligned}
$$

\section{Theorem 3:}

Under $H_{0}, U_{1}, U_{2}, \ldots, U_{n-1}$ are independent.

\section{Proof:}

It is sufficient to show that $U_{i}$ and $U_{i+1}$ are independent. With no loss of generality, we will show that $U_{1}$ and $U_{2}$

First, we observe that the random vector $\boldsymbol{V}_{1.2 .3}=\left(\begin{array}{l}v_{1} \\ v_{2}\end{array}\right) \sim N_{3}\left(\mathbf{0}, \sigma_{v}^{2} I_{3}\right)$.

The set of all possible values of the random vector $\boldsymbol{U}_{1,2}=\left(\begin{array}{l}U_{1} \\ U_{?}\end{array}\right)$ is $A_{1,2}=\left\{\left(\begin{array}{l}0 \\ 0\end{array}\right),\left(\begin{array}{l}1 \\ 0\end{array}\right),\left(\begin{array}{l}0 \\ 1\end{array}\right),\left(\begin{array}{l}1 \\ 1\end{array}\right)\right\}$.

As the multivariate normal distribution $N_{3}\left(\mathbf{0}, \sigma_{v}^{2} \boldsymbol{I}_{3}\right)$ is spherically symmetric about the origin, the random vector $\boldsymbol{U}_{1,2}$ has a uniform distribution over the set $A_{1,2}$, as we will show below.

Based on Theorem 2, we have $P\left(U_{1}=0\right)=P\left(U_{2}=0\right)=0.5$.

The three-dimensional Euclidean space $\mathcal{R}^{3}$ consists of eight octagonal regions, which are given by

$$
\begin{aligned}
& R_{---}=\left\{v_{1}>0, v_{2}>0, v_{3}>0\right\} \\
& R_{--+}=\left\{v_{1}>0, v_{2}>0, v_{3} \leq 0\right\} \\
& \vdots
\end{aligned}
$$


$R_{+++}=\left\{v_{1} \leq 0, v_{2} \leq 0, v_{3} \leq 0\right\}$

$\left(\begin{array}{l}v_{1} \\ v_{2}\end{array}\right)$ has equal probabilities over these eight octagonal regions, i.e., $P\left(\boldsymbol{V}_{1,2,3} \in R_{\mp \mp \mp}\right)=1 / 8$.

in, the vector $\boldsymbol{V}_{1,2,3}=$

This result can also be shown, for example for $P\left(\boldsymbol{V}_{1,2,3} \in R_{---}\right)$, by verifying that

$P\left(\boldsymbol{V}_{1,2,3} \in R_{---}\right)=P\left(v_{1}>0, v_{2}>0, v_{3}>0\right)$

$=P\left(v_{1}>0\right) P\left(v_{2}>0\right) P\left(v_{3}>0\right)$

$=(0.5)(0.5)(0.5)=1 / 8$

Now,

$P\left(U_{1}=0, U_{2}=0\right)=P\left(v_{1} v_{2}>0, v_{2} v_{3}>0\right)$

$=P\left\{\left[\left(v_{1}>0, v_{2}>0\right)\right.\right.$ or $\left.\left(v_{1}<0, v_{2}<0\right)\right],\left[\left(v_{2}>0, v_{3}>0\right)\right.$ or $\left.\left.\left(v_{2}<0, v_{3}<0\right)\right]\right\}$

$=P\left\{\left(v_{2}>0, v_{1}>0, v_{3}>0\right)\right.$ or $\left.\left(v_{2}<0, v_{1}<0, v_{3}<0\right)\right\}$

$=P\left(v_{2}>0, v_{1}>0, v_{3}>0\right)+P\left(v_{2}<0, v_{1}<0, v_{3}<0\right)$

$=P\left(\boldsymbol{V}_{1,2,3} \in R_{---}\right)+P\left(\boldsymbol{V}_{1,2,3} \in R_{+++}\right)$

$=1 / 8+1 / 8$

$=0.25$

$=P\left(U_{1}=0\right) P\left(U_{2}=0\right)$

Similarly, we can show that

$P\left(U_{1}=0, U_{2}=1\right)=P\left(U_{1}=0\right) P\left(U_{2}=1\right)=0.25$

$P\left(U_{1}=1, U_{2}=0\right)=P\left(U_{1}=1\right) P\left(U_{2}=0\right)=0.25$

$P\left(U_{1}=1, U_{2}=1\right)=P\left(U_{1}=1\right) P\left(U_{2}=1\right)=0.25$

Therefore, $U_{1}$ and $U_{2}$ are independent.

\section{Theorem 4:}

Under $H_{0}, U=\sum_{i=1}^{n-1} U_{i} \sim \operatorname{Binomial}(n-1,0.5)$.

Proof:

Based on Theorems 1, 2, and 3, we conclude that $U_{1}, U_{2}, \ldots, U_{n-1}$ are i.i.d. Binomial $(1,0.5)$. Therefore, $U=$ $\sum_{i=1}^{n-1} U_{i} \sim \operatorname{Binomial}(n-1,0.5)$.

Method:

To test the randomness of the ordered residuals we have

$\mathrm{H}_{0}$ : The sample $v_{1}, v_{2}, \ldots, v_{n}$ is random.

$\mathrm{H}_{1}$ : The sample $v_{1}, v_{2}, \ldots, v_{n}$ is not random.

We assume that the value of $U$ that corresponds to a certain sample is $u_{0}$, where $u_{0}=\sum_{i=1}^{n-1} u_{i}$

\section{Copyrights}

Copyright for this article is retained by the author(s), with first publication rights granted to the journal.

This is an open-access article distributed under the terms and conditions of the Creative Commons Attribution license (http://creativecommons.org/licenses/by/4.0/). 